\title{
Fatores parentais associados à atividade física em pré-escolares: a importância da participação dos pais em atividades físicas realizadas pelos filhos
}

\author{
Parental factors associated with physical \\ activity among preschoolers: the \\ importance of parents' participation in \\ physical activities with their children
}

Rev Bras Ativ Fis Saúde p. 205-214 DOI:

http://dx.doi.org/10.12820/rbafs.v.18n2p205

1 Programa Associado de Pós-graduação em Educação Física UPE/UFPB. Grupo de Pesquisa em Estilos de Vida e Saúde, Universidade de Pernambuco, Recife, $P E$, Brasil.

2 Programa de Pós-graduação em Educação Física, Universidade Federal de Santa Catarina, Florianópolis, SC, Brasil.

3 Programa de Pós-graduação em Hebiatria. Grupo de Pesquisa em Estilos de Vida e

Saúde, Universidade de Pernambuco, Recife, PE, Brasil.

Rildo de Souza Wanderley Júnior ${ }^{1}$

Carla Menêses Hardman ${ }^{2}$

Elusa Santina Antunes Oliveira ${ }^{1}$

Anisio Luiz Silva Brito ${ }^{3}$

Simone Storino Honda Barros ${ }^{2}$

Mauro Virgílio Gomes de Barros ${ }^{1,2,3}$

\section{Resumo}

O objetivo desse estudo foi identificar os fatores parentais associados ao baixo nível de atividade física (AF) em crianças pré-escolares. Trata-se de um estudo transversal (base escolar), realizado com uma amostra de 1.042 crianças pré-escolares da cidade do Recife (PE). Um questionário administrado na forma de entrevista face a face com os pais foi utilizado para obtenção de dados demográficos, socioeconômicos, percepção dos pais quanto à importância da $\mathrm{AF}$ para a criança, participação em AF com o filho e nível de AF dos pais e das crianças. A análise dos dados foi realizada mediante regressão logística binária, considerando modelagem hierárquica na entrada das variáveis e o baixo nível de AF da criança como desfecho. Observou-se que $81,9 \%$ dos pais eram fisicamente ativos, enquanto $69,7 \%$ referiram participar de AF com os filhos e a quase totalidade $(99,1 \%)$ dos pais consideraram importante que os filhos participem em AF. O baixo nível de AF foi verificado em 30,3\% das crianças, identificando-se que a participação dos pais em AF com os filhos foi um fator inversamente associado a baixo nível de AF nos pré-escolares $(\mathrm{OR}=0,54 ; \mathrm{IC} 95 \%$ : 0,40-0,73). A prevalência de baixo nível de AF foi significativamente menor entre os filhos de pais que referiram participar de $\mathrm{AF}(26,1 \%$ versus 40,2\%; $\mathrm{p}<0,01)$. Concluiuse que a participação em $\mathrm{AF}$ com os filhos foi o único fator parental associado ao nível de $\mathrm{AF}$ das crianças. Estimular a adoção deste tipo de comportamento pode ser uma estratégia útil na promoção da atividade física infantil, hipótese que precisa ser confirmada em estudo de intervenção.

\section{Palavras-chave}

Atividade física; Pré-escolares; Influência parental.

\begin{abstract}
The aim of this study was to identify parental factors associated with low level of physical activity (PA) among preschoolers. It was a school-based cross-sectional study performed with a sample of 1,042 children from the city of Recife (PE), Brazil. A questionnaire administered by face-to-face interview was used to gather demographic and socioeconomic data, measures of the parental factors (considering important that their children take participation in PA; participating in PA with their children; and, being physically active) and children's level of $P A$. Logistic regression was performed by considering a hierarchical approach for data entry and children's low level of $P A$ as the outcome. It was observed that $81.9 \%$ of the parents were physically actives, $69.7 \%$ reported to participate in $P A$ with their children, and almost all of them referred to consider important that their children take participation in $P A$. The prevalence of low level of $P A$ was $30.3 \%$, lower among preschoolers whose parents reported to participate in $P A$ with their children (26.1\% versus $40.2 \%$; $p<0.01)$. Parents' participation in $P A$ with their children was inversely associated with low level of $P A$ among preschoolers (OR=0.54; 95\%CI 0.40, 0.73). It was concluded that parents' participation in $P A$ with their children was associated with preschoolers' level of PA. Stimulating parents to participate in PA with their children might be useful as a strategy to promote PA during childhood; however this hypothesis needs to be assessed in further studies.
\end{abstract}

\section{Keywords}

Physical activity; Preschoolers; Parental influence. 


\section{INTRODUÇÃO}

Analisar os domínios sociais e culturais da dinâmica familiar para a compreensão da influência dos fatores parentais na prática de atividade física de crianças pré-escolares é de extrema importância para promoção da atividade física nessa população específica. Estudos apontam que a percepção da importância que os pais atribuem à prática de atividades físicas dos filhos ${ }^{1,2}$, a participação em atividades físicas com os filhos ${ }^{3,4}$ e o nível de atividade física dos pais ${ }^{5-8}$ são fatores que têm associação com o nível de atividade física de crianças em idade pré-escolar. Esta é uma linha de investigação importante porque pode auxiliar pesquisadores e profissionais a entenderem as razões para exposição de crianças em idades tão precoces a baixos níveis de atividade física ${ }^{9,10}$.

A prática regular de atividades físicas, nessa fase da vida, tende a repercutir positivamente tanto na saúde física como na saúde psicossocial das crianças ${ }^{11-14}$. Mas apesar do maior reconhecimento quanto aos benefícios imediatos e tardios da prática da atividade física para saúde, grande proporção de crianças pré-escolares está exposta a nível insuficiente de prática de atividades físicas ${ }^{9}$, inclusive no Brasil ${ }^{15}$.

Nesse cenário, alguns modelos teóricos emergem na tentativa de auxiliar na compreensão dos fatores associados à prática de atividades físicas em crianças. $\mathrm{O}$ modelo sociocognitivo proposto por Bandura ${ }^{16}$ sugere que a figura dos pais, servindo ora como modelo ora como elemento de suporte social, e a importância que os pais atribuem ao envolvimento dos filhos com a prática de atividades físicas são fatores que podem exercer uma forte influência no comportamento das crianças quanto à prática de atividades físicas. Entre pesquisadores, o modelo sociocognitivo vem sendo apontado como o mais apropriado para investigação de fatores determinantes da prática de atividades físicas na infância ${ }^{17,18}$.

A importância que os pais atribuem à prática de atividades físicas dos filhos e a participação em atividades físicas com os filhos foram identificados como fatores positivamente associados a maior nível de atividade física em crianças pré-escolares $^{19,20}$. Além disso, o nível de atividade física dos pais é um fator diretamente associado ao nível de atividade física de crianças pré-escolares, resultado que foi observado em estudos adotando delineamento transversal ${ }^{2}$ e de abordagens qualitativas ${ }^{7}$.

Além disso, parece existir independência entre os diferentes fatores parentais que podem exercer influência no nível de atividade física das crianças. Por exemplo, estudo conduzido por Loprinzi et $\mathrm{al}^{3}$ revelou que a importância que os pais atribuem ao envolvimento dos filhos em atividades físicas é um fator independente da efetiva participação dos pais em atividades físicas junto com os filhos.

No Brasil, alguns estudos foram realizados com o objetivo de identificar fatores ambientais e sociais associados ao nível de atividade física de crianças ${ }^{15,21}$. Entretanto, até onde se tem conhecimento nenhum focalizou especificamente na análise de fatores parentais que podem estar associados ao nível de atividade física em crianças pré-escolares. Diante do exposto, o objetivo desse estudo foi identificar os fatores parentais associados ao baixo nível de AF em crianças pré-escolares.

\section{MÉTODOS}

\section{Caracterização do estudo}

Para investigação do problema de pesquisa focalizado neste estudo, recorreu-se a um delineamento transversal e de base escolar, abrangendo componentes descritivos e analíticos. Trata-se de um estudo desenvolvido a partir da análise de dados coleta- 
dos, em 2010, na avaliação inicial (baseline) do Estudo Longitudinal de Observação da Saúde e Bem-Estar da Criança em Idade Pré-escolar (projeto ELOS-Pré). O projeto de pesquisa ao qual este estudo está vinculado foi aprovado pelo Comitê de Ética em Pesquisa com Seres Humanos da Universidade de Pernambuco (CAAE: 0096.0.097.000-10).

\section{População e amostra}

A população alvo deste estudo foi constituída por crianças em idade pré-escolar (3 a 5 anos), matriculadas em escolas de educação infantil das redes pública e privada da cidade de Recife (PE). Segundo dados da Secretaria de Educação do estado de Pernambuco esta população foi estimada, no ano de 2009, a partir do número de matriculas registradas nas Gerências Regionais de Educação (GREs), totalizando 49.338 crianças, distribuídas em 782 escolas de educação infantil.

Considerando os objetivos dos diversos subprojetos que integram o ELOS -Pré, o tamanho amostral foi inicialmente estabelecido para realização de estudo de prevalência (foco da avaliação inicial correspondente ao baseline do estudo longitudinal). Este dimensionamento foi efetuado considerando os seguintes critérios: (a) população alvo de 49.338 crianças; (b) prevalência estimada das variáveis de interesse na população fixada em 50\%; (c) intervalo de confiança de 95\%; (d) erro máximo tolerável de quatro pontos percentuais; e (e) efeito do delineamento amostral estabelecido em 1,5 pontos $($ deff $=1,5)$ devido ao recurso de amostragem por conglomerados. Assim, o tamanho inicial da amostra foi estimado em 890 participantes, acrescentando-se mais $20 \%$ a fim de lidar com possíveis perdas e recusas, o que resultou numa amostra com 1.068 crianças.

Considerando o número médio de crianças matriculadas nas escolas de educação infantil (38,5 crianças/escola), estabeleceu-se que a coleta de dados deveria ser efetuada em 28 escolas (unidades amostrais) a fim de que a dimensão amostral desejada $(n=1.068)$ pudesse ser alcançada. A amostra foi selecionada através de um procedimento de amostragem por conglomerados em um único estágio, considerando a escola como unidade amostral. Todas as escolas de educação infantil na Cidade do Recife foram consideradas elegíveis para inclusão no estudo. Visando garantir maior representatividade, adotou-se como critério de estratificação a proporcionalidade de escolas de educação infantil segundo tipo (pública ou privada), porte e localização nas seis regiões político-administrativas (RPA) da cidade do Recife. Foram classificadas como de pequeno porte as escolas com menos de 50 crianças matriculados na educação infantil; de médio porte, aquelas com 50 a 199 crianças matriculadas; e, de grande porte, aquelas com 200 ou mais crianças matriculadas neste nível de ensino.

O sorteio das escolas participantes foi efetuado considerando uma lista numerada com o nome de todas as escolas elegíveis para a participação no estudo. Para a realização do sorteio, utilizou-se o programa EpiInfo 6 para geração de números aleatórios. A unidade amostral final foi representada pela turma, mas o elemento amostral (unidade de análise) foi a criança. Todas as crianças matriculadas na escola foram consideradas elegíveis e convidadas a participar do estudo. No desenvolvimento do presente estudo foram excluídas somente as crianças que estavam ausentes da escola no período de realização da coleta de dados.

\section{Coleta de dados}

Os dados foram coletados no período de agosto a novembro de 2010 por uma equipe previamente treinada e composta por estudantes de graduação e de pós- 
graduação (mestrado e doutorado), todos seguindo um protocolo de procedimentos operacionais previamente padronizados a fim de orientar a coleta de dados. Todo o trabalho de campo foi diretamente supervisionado pelos pesquisadores envolvidos no desenvolvimento do projeto.

Para a coleta de dados foi utilizado um questionário respondido pelos pais ou responsáveis legais das crianças mediante entrevista face a face. $\mathrm{O}$ instrumento foi previamente testado em estudo piloto que consistiu de aplicações repetidas do instrumento a um grupo de 65 pais, adotando-se um intervalo de sete dias entre as aplicações. Os resultados do estudo piloto indicaram bons indicadores de reprodutibilidade para medidas de dados demográficos e socioeconômicos (coeficientes de correlação de Spearman [rho] superiores a 0,80), assim como para medida da atividade física $(\mathrm{rho}=0,83)^{22}$. Para medida do nível de atividade física dos pais foi utilizado a versão curta do questionário internacional de atividades físicas (IPAQ, versão curta) ${ }^{23}$.

\section{Variáveis}

As variáveis independentes foram três fatores parentais que, segundo a teoria sociocognitiva ${ }^{16}$, podem exercer influência no comportamento das crianças quanto à prática de atividades físicas. A primeira variável foi definida como a importância que os pais atribuem ao envolvimento dos filhos com a prática de atividades físicas, sendo determinada pela pergunta: $\mathrm{O}(\mathrm{A})$ senhor(a) considera importante que o(a) seu(sua) filho(a) participe de brincadeiras, jogos ou práticas esportivas? A segunda variável foi a participação dos pais em atividades físicas com o filho, aferida pela pergunta: $\mathrm{O}(\mathrm{A})$ senhor(a) participa de brincadeiras, jogos ou praticas esportivas com o(a) seu(sua) filho(a)? A primeira questão possuía três possibilidades de resposta: "sim", "não" e "não sabe informar"; e a segunda duas possibilidades de resposta: "sim" e "não". Por fim, considerou-se também o nível de atividade física dos pais que foi classificado em duas categorias (insuficientemente ativos e ativos), adotando-se a prática de, no mínimo, 150 minutos/semana em atividades físicas de intensidade moderada a vigorosa como critério para definir que o sujeito era fisicamente ativo.

A variável dependente foi o nível de atividade física da criança, adotando-se como desfecho o "baixo nível de atividade física". A medida foi obtida considerando o relato dos pais quanto ao tempo despendido pelas crianças em jogos e brincadeiras ao ar livre nos três períodos do dia (manhã, tarde e noite), tanto em um dia típico de semana (segunda à sexta) quanto do final de semana (sábado e domingo). Detalhamento das perguntas e categorias de resposta está apresentada no estudo de Oliveira et $\mathrm{a}^{22}$.

O tempo relatado pelos pais em cada um destes seis períodos de referência foi registrado considerando cinco escores numéricos e respectivas categorias de resposta: $0=0$ minuto; $1=1-15$ minutos; $2=16-30$ minutos; $3=31-60$ minutos; $\mathrm{e}$ $4=$ mais de 60 minutos. Assim, para cada criança os pais relataram seis estimativas de tempo despendido em atividades físicas típicas da idade, sendo três relativas ao dia de semana e três relativas ao dia de final de semana típico (habitual). Em seguida, efetuou-se cálculo de um escore global expressando o nível de atividade física da criança, com amplitude de variação de 0 a 24 pontos (seis períodos de referencia $\mathrm{X}$ escore numérico atribuído ao tempo relatado pelos pais). Por fim, este escore global foi dicotomizado, classificando-se as crianças classificadas no quartil inferior da escala como casos de "baixo nível de atividade física".

Além das variáveis independentes e dependentes, considerou-se também um conjunto de fatores que poderiam confundir a análise de associação entre fatores 
parentais e nível de atividade física dos pré-escolares, conforme ilustrado na Figura 1 que apresenta o modelo teórico de determinação da atividade física infantil. Além do sexo e idade das crianças, foram analisadas ainda variáveis socioeconômicas: nível socioeconômico, estabelecido a partir da renda familiar dicotomizada em dois salários mínimos (<2 e $\geq 2$ salários mínimos); número de filhos (1, 2 e 3 ou mais filhos); e escolaridade materna que foi dicotomizada considerando o ensino médio completo ou superior como critério de referência. Foram considerados ainda dois fatores escolares: tipo de escola (pública e privada) e turno de matrícula na escola de educação infantil (manhã e tarde). A presença de excesso de peso na criança foi determinada mediante cálculo do índice de massa corporal e classificação por meio dos pontos de corte sugeridos por Conde e Monteiro ${ }^{24}$.

\section{Análise de dados}

A análise de dados foi realizada utilizando o programa SPSS (versão 10). A análise descritiva foi efetuada mediante distribuição de frequências e cálculo de intervalos de confiança (IC95\%) para proporções. O teste de Qui-quadrado foi usado para testar a associação bivariável entre as variáveis independentes e o nível de atividade física dos pré-escolares.

Verificação da interação entre os fatores parentais e as covariáveis (fatores de confusão) foi efetuada previamente às análises multivariáveis, mas nenhum efeito de interação foi observado. A análise multivariável foi realizada para verificar a associação entre as variáveis independentes (fatores parentais) e o nível de atividade física dos pré-escolares com ajustamento para potenciais fatores de confusão. A determinação da ordem de entrada das variáveis independentes foi efetuada hierarquicamente em duas etapas, seguindo o modelo de análise apresentado na Figura 1. Na primeira etapa foram introduzidos os fatores demográficos e socioeconômicos que se encontram no mesmo nível hierárquico do modelo e, na segunda etapa, os demais fatores, inclusive os parentais que foram definidos operacionalmente como variáveis independentes no presente estudo. Da primeira para a segunda etapa foram mantidas somente aquelas variáveis que apresentaram associação com o desfecho equivalente a um valor $p$ inferior a 0,20 .

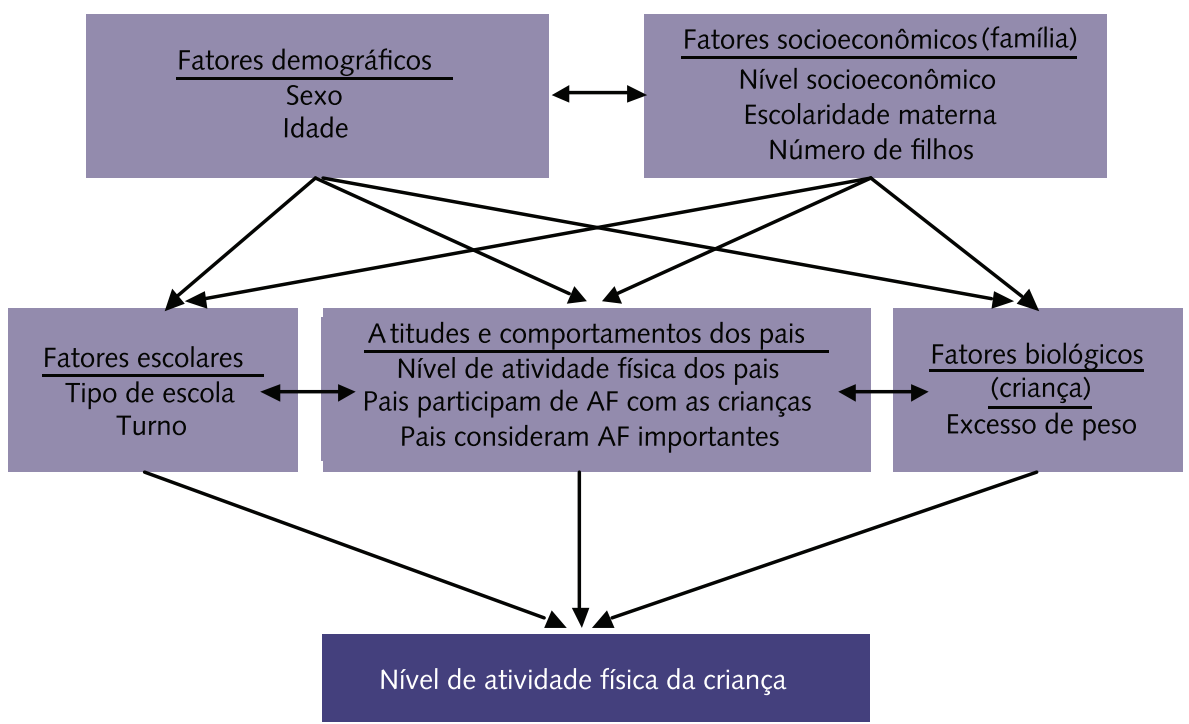

Figura 1 - Modelo teórico de determinação do nível de atividade física em crianças pré-escolares. 


\section{RESULTADOS}

Nas 28 escolas sorteadas para realização do estudo, identificou-se que havia 1.155 crianças matriculadas, sendo que destas 22 tinham idade inferior $(n=9)$ ou superior $(n=13)$ à faixa etária definida como critério para inclusão no estudo (3 a 5 anos). Além disso, outras 91 crianças não foram incluídas por recusa dos pais em participar do estudo ou porque as crianças estiveram ausentes da escola durante o período de coleta de dados. Participaram do estudo 1.042 crianças, um tamanho amostral que permite detectar como significativas razões de chances (OR) iguais ou superiores 1,45 , considerando intervalo de confiança de $95 \%$, poder estatístico de $80 \%$ e prevalência do desfecho de 30\% entre não expostos e de 38,3\% entre expostos.

As características das crianças incluídas no estudo estão apresentadas na Tabela 1. A média de idade foi de 4,3 anos ( $\mathrm{DP}=0,8)$. Não foram observadas diferenças estatisticamente significativas entre meninos e meninas em relação a nenhuma das variáveis independentes, exceto em relação ao turno de matricula na pré-escola.

Tabela 1 - Características dos participantes em relação aos fatores demográficos, socioeconômicos e parentais, estratificada por sexo.

\begin{tabular}{|c|c|c|c|c|}
\hline Variável & Categorias & Meninos & Meninas & Todos \\
\hline \multirow[t]{3}{*}{ Idade (anos) } & 3 & $107(18,9 \%)$ & $84(15,6 \%)$ & $191(17,3 \%)$ \\
\hline & 4 & $181(32,0 \%)$ & $161(29,9 \%)$ & $342(31,0 \%)$ \\
\hline & 5 & $278(49,1 \%)$ & $293(54,5 \%)$ & $571(51,7 \%)$ \\
\hline \multirow[t]{2}{*}{ Renda familiar (salários mínimos) } & $<2$ & $79(52,0 \%)$ & $669(75,4 \%)$ & $748(72,0 \%)$ \\
\hline & $\geq 2$ & $73(48,0 \%)$ & $218(24,6 \%)$ & $291(28,0 \%)$ \\
\hline \multirow{2}{*}{$\begin{array}{l}\text { Escolaridade materna (ensino médio } \\
\text { completo ou superior) }\end{array}$} & Não & $39(35,1 \%)$ & $380(42,7 \%)$ & $419(41,9 \%)$ \\
\hline & Sim & $72(64,9 \%)$ & $510(57,3 \%)$ & $582(58,1 \%)$ \\
\hline \multirow[t]{3}{*}{ Número de filhos } & 1 & $47(30,1 \%)$ & $298(33,0 \%)$ & $345(32,6 \%)$ \\
\hline & 2 & $59(37,8 \%)$ & $330(36,6 \%)$ & $389(36,8 \%)$ \\
\hline & $\geq 3$ & $50(32,1 \%)$ & $274(30,4 \%)$ & $324(30,6 \%)$ \\
\hline \multirow[t]{2}{*}{ Tipo de escola } & Pública & $247(43,6 \%)$ & $244(45,4 \%)$ & $491(44,5 \%)$ \\
\hline & Privada & $319(56,4 \%)$ & $294(54,6 \%)$ & $613(55,5 \%)$ \\
\hline \multirow[t]{2}{*}{ Turno } & Manhã & $288(50,9 \%)$ & $241(44,8 \%)$ & $529(47,9 \%)$ \\
\hline & Tarde & $278(49,1 \%)$ & $297(55,2 \%)$ & $575(52,1 \%)$ \\
\hline \multirow[t]{2}{*}{ Criança com excesso de peso } & Não & $432(80,7 \%)$ & $356(70,2 \%)$ & $788(75,6 \%)$ \\
\hline & Sim & $103(19,3 \%)$ & $151(29,8 \%)$ & $254(24,4 \%)$ \\
\hline \multirow[t]{2}{*}{ Pais participam de AF com os filhos } & Não & $36(22,9 \%)$ & $284(31,6 \%)$ & $320(30,3 \%)$ \\
\hline & Sim & $121(77,1 \%)$ & $615(68,4 \%)$ & $736(69,7 \%)$ \\
\hline \multirow{2}{*}{$\begin{array}{l}\text { Pais consideram importante que os } \\
\text { filhos participem de AF }\end{array}$} & Não & $0(0 \%)$ & $10(1,1 \%)$ & $10(0,9 \%)$ \\
\hline & Sim & $156(100 \%)$ & $891(98,9 \%)$ & $1047(99,1 \%)$ \\
\hline \multirow{2}{*}{ Pais fisicamente ativos } & Não & $34(22,2 \%)$ & $152(17,4 \%)$ & $186(18,1 \%)$ \\
\hline & Sim & $119(77,8 \%)$ & $720(82,6 \%)$ & $839(81,9 \%)$ \\
\hline
\end{tabular}

Baixo nível de atividades físicas (quartil inferior do escore global expressando nível de atividade física habitual) foi verificado em 30,3\% (IC95\%:27,6-33,2) das crianças. A prevalência de baixo nível de $\mathrm{AF}$ foi significativamente menor entre os filhos de pais que referiram participar das atividades físicas (26,1\% versus $40,2 \%)$, conforme apresentado na Figura 2. 


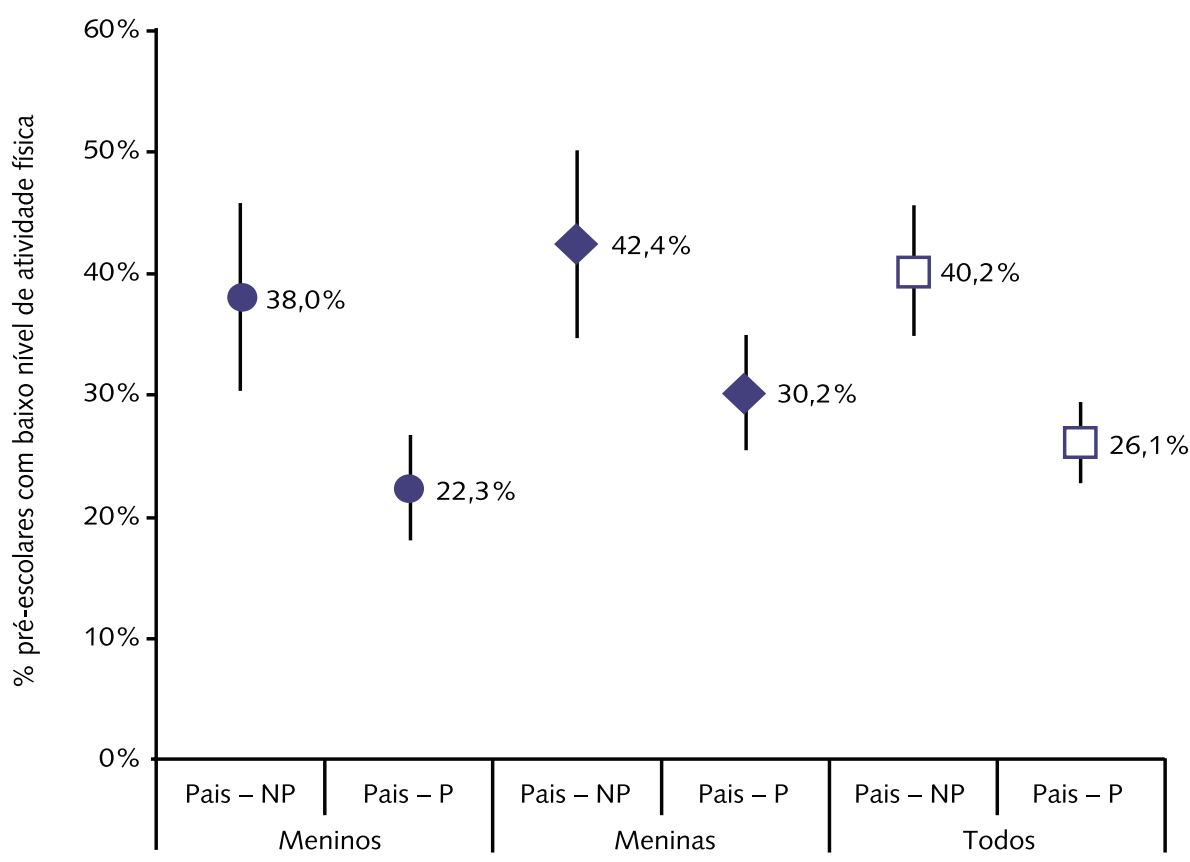

Figura 2 - Proporção de crianças pré-escolares com baixo nível de atividade física segundo participação dos pais em atividades físicas (jogos e brincadeiras) com os filhos, por sexo.

Pais - NP= pais que referiram não participar de AF com os filhos; Pais - $\mathrm{P}=$ pais que referiram participação em AF com os filhos.

$\mathrm{Na}$ Tabela 2 estão os resultados da regressão logística binária entre os fatores parentais (variáveis independentes) e o baixo nível de atividade física das crianças pré-escolares (desfecho). Observa-se que somente a participação dos pais em atividades físicas com os filhos foi identificada como fator inversamente associado ao baixo nível de atividade física das crianças pré-escolares.

Tabela 2 - Razões de chances (OR) e respectivos intervalos de confiança (IC95\%) para identificação de fatores parentais associados ao baixo nível de atividade física em crianças pré-escolares.

\begin{tabular}{lcc}
\hline Fator parental & $\begin{array}{c}\text { OR bruto } \\
(I C 95 \%)\end{array}$ & OR ajustado* (IC95\%) \\
\hline Pais fisicamente ativos & $\begin{array}{c}0,85(0,60-1,19) \\
\mathrm{P}=0,33\end{array}$ & $\begin{array}{c}0,84(0,59-1,19) \\
\mathrm{P}=0,32\end{array}$ \\
\hline Pais participam de atividades físicas & $0,52(0,40-0,69)$ & $0,52(0,39-0,69)$ \\
com os filhos & $\mathrm{P}<0,01$ & $\mathrm{P}<0,01$ \\
\hline Pais consideram importante que os & $1,02(0,26-3,96)$ & $1,59(0,31-8,18)$ \\
filhos participem de atividades físicas & $\mathrm{P}=0,98$ & $\mathrm{P}=0,58$ \\
\hline
\end{tabular}

O ajuste foi realizado pelas seguintes covariáveis: idade e sexo das crianças; renda familiar; escolaridade materna; número de filhos; tipo de escola; turno e excesso de peso da criança.

\section{DISCUSSÃO}

O objetivo desse estudo foi identificar fatores parentais associados ao baixo nível de atividade física em crianças pré-escolares. Os principais achados foram: (1) a identificação de que a participação dos pais em atividades físicas com os filhos é um fator inversamente associado ao baixo nível de atividade física neste grupo de pré-escolares; e (2) o nível de atividade física dos pais não apresentou associação com o nível de atividade física das crianças.

Entretanto, tais achados precisam ser interpretados e aplicados com cautela. Admite-se a possibilidade de causalidade reversa, tendo em vista o uso de dados 
provenientes de um estudo transversal. A participação dos pais em atividades físicas realizadas com os filhos pode ser, na verdade, uma consequência e não uma causa do maior nível de atividade física apresentado pelas crianças. Outras possíveis limitações são os vieses de resposta e classificação, tendo em vista que a variável de desfecho (baixo nível de atividade física das crianças) foi uma medida referida pelos pais que não acompanham integralmente a rotina diária das crianças. No entanto, esta medida de aproximação foi testada em estudo de validação conduzido por Burdette, Whitaker e Daniels ${ }^{25}$ e mostrou boa correlação com a medida objetiva da atividade física em pré-escolares.

A observação de associação inversa entre a participação dos pais em atividades físicas com os filhos e o baixo nível de atividade física das crianças é um resultado convergente em relação aos achados apresentados em outros estudos transversais ${ }^{3,5,26,27}$. Tal associação foi identificada em estudos que utilizaram instrumentos distintos para mensuração do nível de atividade física das crianças, abrangendo investigações que utilizaram observação $\operatorname{direta}^{26}$, acelerometria ${ }^{3} \mathrm{e}$ instrumentos do tipo papel-caneta ${ }^{28}$. Esta convergência de resultados, que independe do método usado para medida da atividade física, foi confirmada em recente metanálise que não identificou uma diferença entre o poder das associações entre fatores parentais e o nível de atividade física de pré-escolares determinado mediante utilização de medidas diretas ou referidas ${ }^{8}$.

Crianças em idade pré-escolar não apresentam autonomia para escolhas relacionadas à prática de atividades físicas, assim, é de se esperar que a participação dos pais constitua um fator com forte influência no nível de atividade física das crianças. Diante destes achados e independente das limitações inerentes aos delineamentos de natureza transversal, a participação dos pais em atividades físicas com os filhos vem sendo considerada uma importante estratégia no planejamento de intervenções para promoção de um estilo de vida fisicamente ativo em crianças ${ }^{7,28}$.

Diferente do que está relatado em outros estudos ${ }^{5-7}$, não foi observada associação entre o nível de atividade física dos pais e o nível de atividade física das crianças. Uma possível explicação para tal divergência pode ser o método utilizado para efetuar a medida da atividade física nos adultos. Tendo em vista que a maior parte dos estudos onde esta associação foi observada, empregava acelerometria para medida da atividade física ${ }^{3,6,7}$. Somente no estudo de Spurrier et $\mathrm{al}^{2}$, que utilizaram questionários para medida da atividade física dos pais, observou-se associação entre o nível de atividade física dos pais e das crianças.

Também em discordância em relação aos achados de outros estudos ${ }^{3,19}$, não foi verificada associação entre a importância que os pais atribuem à participação dos filhos em atividades físicas com o nível de atividade física efetivamente aferido nas crianças pré-escolares. Por outro lado, esse resultado está em consonância com o que foi relatado por Loprinzi e Trost ${ }^{3}$ que também identificaram que esta variável parental não estava correlacionada ao nível de atividade física das crianças.

Os achados aqui apresentados sugerem que o êxito dos programas de promoção da atividade física para crianças pré-escolares pode estar intimamente relacionado ao suporte oferecido pelos pais aos filhos no tocante à prática de atividades físicas. Mais especificamente, a participação dos pais em atividades físicas realizadas pelos filhos parece ser um comportamento importante e uma estratégia potencialmente efetiva para promoção de estilos de vida fisicamente mais ativos na infância ${ }^{29}$. Entretanto, a inexistência de resultados derivados de estudos de intervenção constitui uma importante lacuna de conhecimento e deverá ser superada em investigações futuras. Estudos com abordagem qualitativa de investigação, a exemplo 
da investigação conduzida por Dwyer et $\mathrm{al}^{30}$, poderão auxiliar a compreensão dos mecanismos pelos quais as atitudes e comportamentos dos pais podem influenciar positivamente a conduta das crianças em relação às atividades físicas.

\section{Agradecimentos}

Estudo apoiado com auxílio financeiro da Coordenação de Aperfeiçoamento de Pessoal de Nível Superior e da Fundação de Amparo à Ciência e Tecnologia do Estado de Pernambuco.

\section{REFERÊNCIAS}

1. Irwin JD, He M, Bouck LM, Tucker P, Pollett GP. Preschoolers' physical activity behaviors: Parents' perspectives. Can J Public Health. 2005;96:299-305.

2. Spurrier NJ, Magarey AA, Golley R, Curnow F, Sawyer MG. Relationships between the home environment and physical activity and dietary patterns of preschool children: a cross-sectional study. In J Behav Nutr Phys Act. 2008;5:1-12.

3. Loprinzi PD, Trost SG. Parental influences on physical activity behavior in preschool children. Prev Med. 2010;50:129-133.

4. Oliver AM, Schofield GM, Schluter PJ. Parent influences on preschoolers' objectively assessed physical activity. J Sci Med Sport. 2010;13:403-409.

5. Hinkley T, Crawford D, Salmon J, Okely AD, Hesketh K. Preschool Children and Physical Activity: A Review of Correlates. Am J Prev Med. 2008;34:435-441.

6. Alderman BL, Benham-Deal TB, Jenkins JM. Change in parental influence on children's physical activity over time. J Phys Act Health. 2010;7:60-67.

7. Tucker P, Van Zandvoort MM, Burke SM, Irwin JD. The influence of parents and the home environment on preschoolers' physical activity behaviours: A qualitative investigation of childcare providers' perspectives. BMC Public Health 2011; 11; 1: 168.

8. Mitchell J, Skouteris H, McCabe M et al. Physical activity in young children: a systematic review of parental influences. E Child Devel Care. 2011;182:1411-1437.

9. Cardon GM, De Bourdeaudhuij IM. Are preschool children active enough? Objectively measured physical activity levels. Res Q Exerc Sport. 2008;79:326-332.

10. Telama R. Tracking of physical activity from childhood to adulthood: a review. Obes Facts. 2009;2:187-195.

11. Vale SMCG, Santos RMR, Soares-Miranda LMC. Objectively measured physical activity and body mass index in preschool children. Inter J Pedi. 2010;0:1-6.

12. Janz KF, Gilmore JME, Levy SM. Physical activity and femoral neck bone strength during childhood: the Iowa Bone Development Study. Bone. 2007;41:216-222.

13. Matvienko O, Ahrabi-Fard I. The effects of a 4-week after-school program on motor skills and fitness of kindergarten and first-grade students. Am J Health Promot. 2010;24:299-303.

14. Tomporowski PD, Lambourne K, Okumura MS. Physical activity interventions and children's mental function: An introduction and overview. Prev Med. 2011;52:S3-S9.

15. Barros SSH, Lopes AS, Barros MVG. Prevalência de baixo nível de atividade física em crianças pré-escolares. Rev Bras Cineantropom Desempenho Hum. 2012;14:390-400.

16. Bandura A. Health Promotion by Social Cognitive Means. Health Educ Behav. 2004;31:143-164.

17. Nyberg G, Sundblom E, Norman A, Elinder LS. A healthy school start - Parental support to promote healthy dietary habits and physical activity in children: Design and evaluation of a cluster-randomised intervention. BMC Public Health. 2011;11:185.

18. Baranowski TPC, Parcel GS. Health behaviour and health education Theory, research, and practice. San Francisco-Jossey-Bass, 2002.

19. Aarts MJ, Wendel-Vos W, van Oers HAM, Van De Goor IAM, Schuit AJ. Environmental determinants of outdoor play in children: a large-scale cross-sectional study. Am J Prev Med. 2010;39:212-219.

20. Allender S, Cowburn G, Foster C. Understanding participation in sport and physical activity among children and adults: a review of qualitative studies. Health Educ Res. 2006; 21:826-835.

21. Gaya A, Torres L. Hábitos de vida de escolares de uma escola da rede municipal de Porto Alegre. Revista Perfil- UFRGS 1997;1:24-37. 
22. Oliveira NKR, Lima RA, Mélo EM, Santos CM, Barros SSHB, Barros MVGB. Reprodutibilidade de questionário para medida da atividade física e comportamento sedentário em crianças pré-escolares. Rev Bras Ativ Fis Saud. 2011;16:228-233.

23. Matsudo S, Timóteo A, Matsudo V, Douglas A, Erinaldo A, Oliveira LC, et al. Questionário Internacional de Atividade Física (IPAQ): Estudo de validade e reprodutibilidade no Brasil. Rev Bras Ativ Fis Saud. 2001;6(2):5-18.

24. Conde WL, Monteiro CA. Valores críticos do índice de massa corporal para classificação do estado nutricional de crianças e adolescentes brasileiros. J Pediatr (Rio J). 2006;82(4):266-72.

25. Burdette HL, Whitaker RC, Daniels SR. Parental report of outdoor playtime as a measure of physical activity in preschool-aged children. Arch Pediatr Adol Med. 2004;58:353-7.

26. Klesges RC, Eck LH, Hanson CL, Haddock CK, Klesges L. Effects of obesity, social interactions, and physical environment on physical activity in preschoolers. Health Psychol. 1990;9:435-449.

27. Zecevic CA, Tremblay L, Lovsin T, Michel L. Parental influence on young children's Physical Activity. Int J Pediatr . 2010:1-9.

28. Golley RK, Hendrie GA, Slater A, Corsini N. Interventions that involve parents to improve children's weight-related nutrition intake and activity patterns - what nutrition and activity targets and behavior change techniques are associated with intervention effectiveness? Obes Rev. 2011;12:114-130.

29. Uijtdewilligen L, Nauta J, Singh AS. Determinants of physical activity and sedentary behaviour in young people: a review and quality synthesis of prospective studies. Brit J Sport Med. 2011;45:896-905.

30. Dwyer GM, Higgs J, Hardy LL, Baur LA. What do parents and preschool staff tell us about young children's physical activity: A qualitative study. Inter J Behav Nutr Phys Act 2008;5:66-76.

Endereço para Correspondência Rua Arnóbio Marques, 310, Campus Universitário HUOC/ESEF Santo Amaro, Recife - PE, 50100-130 E-mail: rildowanderley.esef@gmail.com Telefone: +55 81 3183-3376
Recebido 30/01/2013

Revisado 13/02/2013

Aprovado 06/05/2013 